\title{
So müssen ab sofort die vieldiskutierten Terminservicestellen funktionieren
}

_ Die KBV hat jetzt mit dem GKV-Spitzenverband vereinbart, wie genau die Servicestellen für die Vermittlung von Facharztterminen eingerichtet werden. Dazu wurde eine Anlage 28 des Bundesmantelvertrags-Ärzte beschlossen. Laut Gesetz müssen die Servicestelle interessierten Patienten innerhalb einer Woche einen Termin bei einem niedergelassenen Facharzt oder einer Einrichtung nach $\$ 95$ Abs. 1 Satz 1 SGB V (z. B. in einem Krankenhaus) vermitteln. Dieser muss spätestens vier Wochen, nachdem der Patient bei der Servicestelle seinen Wunsch vorgetragen hat, stattfinden.

Schafft es die Servicestelle nicht innerhalb der ersten Woche, einen Termin bei einem Vertragsarzt zu vermitteln, muss sie dem Patienten in der darauf folgenden Woche einen Termin in einem Krankenhaus anbieten. Die Wartezeit für den Versicherten verlängert sich dann um eine weitere Woche. Bei Routineuntersuchungen und Bagatellfällen bleibt es bei der im Gesetz bereits geregelten angemessenen Wartezeit.

\section{MMW-KOMMENTAR}

Der überweisende Vertragsarzt muss gemäß § 4 Abs. 2 der neuen Vereinbarung direkt auf der Überweisung deutlich macht, ob es sich um eine verschiebbare Routineuntersuchung bzw. eine Bagatellerkrankung handelt oder ob die neuen Fristen einzuhalten sind. Wie genau dies gekennzeichnet werden soll, müssen nun die einzelnen KVen regeln, die also ein gewisses Ermessen haben, welche Fälle gekennzeichnet werden müssen.

In $\$ 5$ der Vereinbarung ist geregelt, dass der Versicherte keinen Anspruch darauf hat, dass ihm die Terminservicestelle einen Termin bei seinem Wunsch-Arzt vermittelt. Sollte der Versicherte der Servicestelle sofort oder sehr schnell mitteilen, dass er einen angebotenen Termin nicht wahrnehmen kann, soll ihm ein weiterer angeboten werden wenn die Servicestelle denn über einen verfügt.

Die zumutbare Entfernung wird in $\S 6$ definiert. Für die Arztgruppen der allgemeinen fachärztlichen Versorgung (z. B. HNO, Gynäkologie, Augenheilkunde) nimmt man die Zeit, die der Patient bis zum nächsten erreichbaren geeigneten Facharzt brauchen würde, plus maximal 30 Minuten. Ein Arzt aus der spezialisierten und gesonderten fachärztlichen Versorgung (z. B. Kardiologen, Pneumologen, Gastroenterologen) darf maximal 60 Minuten weiter weg sein als sein nächster Kollege. Diese Vorgaben, die auch für die Behandlung im Krankenhaus gelten, dürfen die KVen auch durch Kilometerangaben ersetzen. Darüber hinaus soll die Terminservicestelle auch die individuelle Mobilität des Versicherten, die besonderen örtlichen Verhältnisse sowie die öffentliche Verkehrsanbindung berücksichtigen. Diese Vorgaben stehen auch in der Gesetzesbegründung; die KVen haben bei der Umsetzung einen Ermessensspielraum.

Gerade weil „,weniger dringende" Fälle besonders gekennzeichnet werden müssen, kommt auf die Hausärzte wohl eine weitere bürokratische Belastung zu. Selbst veranlasste Überweisungen aus medizinischen Gründen werden sie wohl grundsätzlich nicht kennzeichnen. Doch was ist mit Überweisungen, die vom Patienten gewünscht werden? Schließlich hat der Patient freie Arztwahl und kann einen Facharzt auch ohne Überweisung aufsuchen. Bekommt er dort keinen Termin, wird er sich sicherlich an den Hausarzt wenden und eine nicht gekennzeichnete Überweisung wünschen. Vonseiten der Facharztpraxen wird dann eventuell verlangt, wegen eines bevorzugten Termins anzurufen, wie wir es bereits heute beobachten können. Woher die Hausarztpraxen die Zeit für solche Telefonate nehmen sollen, wurde von den Machern dieser Regelung offensichtlich nicht berücksichtigt!

\section{Hier steht eine Anzeige.}

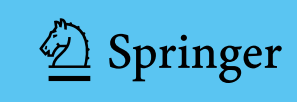

